\title{
Overexpression of PDZK1IP1, EEF1A2 and RPL41 genes in intrahepatic cholangiocarcinoma
}

\author{
GUANGHUA YANG $^{1,2}$ and HUAJIE $\mathrm{ZONG}^{1}$ \\ ${ }^{1}$ Department of General Surgery, Huashan Hospital Affiliated to Fudan University, Shanghai 200040; \\ ${ }^{2}$ The First Department of General Surgery, The Seventh People's Hospital Affiliated to Shanghai University \\ of Traditional Chinese Medicine, Shanghai 200137, P.R. China
}

Received May 8, 2015; Accepted February 24, 2016

DOI: $10.3892 / \mathrm{mmr} .2016 .5110$

\begin{abstract}
Intrahepatic cholangiocarcinoma (iCCA) is an aggressive malignancy in the liver, which is associated with a poor prognosis. However, the molecular pathogenesis of iCCA remains unclear. RNA-Seq for tumor and para-tumor sample pairs enables the characterization of changes in the gene expression profiles of patients with iCCA. The present study analyzed RNA-Seq data of seven iCCA para-tumor and tumor sample pairs. Differential gene expression analysis demonstrated significant upregulation of PDZK1IP1, EEF1A2 and RPL41 (ENSG00000279483) genes in the iCCA samples when compared with the matched para-tumor samples. Furthermore, genes associated with the immune system, metabolism and metabolic energy were significantly downregulated in the iCCA tumor tissues, indicating that this is involved in the pathogenesis of iCCA. The present study aimed to elucidate the gene expression patterns associated with the tumorigenesis of iCCA by comparing tumor and normal tissues, in order to isolate novel diagnostic factors for iCCA.
\end{abstract}

\section{Introduction}

Intrahepatic cholangiocarcinoma (iCCA) is the second most common type of aggressive malignancy of the liver, resulting in a poor prognosis (1). Over the past two decades, the incidence of iCCA has increased by $22 \%$, and iCCA is associated with high rates of mortality with $<10 \%$ of patients surviving 5 years following diagnosis (2) due to its rapid growth and tendency to invade adjacent organs and metastasize. However, the molecular pathogenesis of iCCA remains to be elucidated.

Genome-wide analysis of gene expression with microarray and RNA-Seq technology has enabled an overall insight into

Correspondence to: Dr Huajie Zong, Department of General Surgery, Huashan Hosptial Affiliated to Fudan University, 12 Middle Urumqi Road, Shanghai 200040, P.R. China

E-mail: zonghuajie2004@aliyun.com

Key words: EEF1A2, RPL41, intrahepatic cholangiocarcinoma, RNA-seq
iCCA molecular processes, and will enable characterization of the complex mechanism underlying the development of iCCA. Numerous microarray studies have identified hundreds of differentially expressed genes (DEGs) in tumor tissues or cell lines of iCCA when compared with normal intrahepatic bile duct epithelia or normal liver tissues $(3,4)$, and a series of genes have been revealed as potential biomarkers or therapeutic targets for iCCA. A recent RNA-Seq study determined fusion and mutation in iCCA para-tumor/tumor sample pairs (5), allowing the comparison of gene expression in tumor samples of iCCA.

The present study analyzed RNA-Seq data from seven para-tumor and tumor sample pairs of iCCA. In order to clarify the transcriptomic difference between tumor and para-tumor tissue of iCCA, DEG analysis was performed. The RPL41 gene has two ensemble ID (ENSG00000279483 and ENSG00000229117) in the latest release of the Ensembl GRCh38 human reference genome. In the present analysis, only the differential expression of ENSG00000279483 was identified. It was identified that the expression of PDZK1IP1, EEF1A2 and RPL41 genes was significantly upregulated in the iCCA samples, this may indicate that PDZK1IP1, EEF1A2 and RPL41 are key molecular markers associated with the tumorigenesis and progression of iCCA. Notably, RPL41 has been annotated in the Genome Reference Consortium (GRC)h38 database for the first time and is also identified to be overexpressed in iCCA. Furthermore, genes associated with the immune system, metabolism and metabolic energy were significantly downregulated in iCCA tumor tissues, which indicates that the basic functions of the liver and cholangiole was affected in iCCA. The present study provided a global gene expression view of the tumorigenesis of iCCA.

\section{Materials and methods}

RNA-Seq data processing and expression profiling. Seven para-tumor/tumor pairs of iCCA RNA-Seq data were downloaded from the NCBI Gene Expression Omnibus (GEO; accession no. GSE63420; http://www.ncbi.nlm.nih.gov/geo/) and subsequently reanalyzed. These data were obtained from fresh frozen tumour tissue and corresponding normal tissue from 7 resected patients with iCCA, which were collected from the Biorepository Tissue Bank at the Icahn School of Medicine at Mount Sinai (New York, NY, USA) (5). Data were generated by the Illumina HiSeq 2500 system with 100 nucleotide 
single-end reads. High-quality sequences were aligned to Ensembl human genome GRCh38 (released on Jul 2014), which contain 20,364 coding genes and 196,345 gene transcripts, using a TopHat (v2.0.13) alignment tool with default parameters (6). Gene/transcripts expression levels were quantified by Cufflinks (v2.0.2) (7), yielding raw read counts and normalized FPKM values (reads per kilobase per million reads) which enables the comparison between para-tumor and tumor samples. Then, the gene/transcript expression values were $\log 2$ transformed and genes with null values were removed in all 7 pairs of samples. Pearson correlation coefficient and hierarchical clustering analysis (HCA), calculated by the Ward method (8) based on a distance matrix of the Pearson correlation of the samples, were performed in the R (www.r-project.org/) environment using its 'base' function and 'stat' packages. Samples with similar gene expression profiles were clustered together.

Differential expression analysis. Differential expression of genes between tumor samples and matched para-tumor samples was identified. Cuffdiff (v2.0.2) was applied to calculate fold changes using the FPKM value of each gene between tumor and matched para-tumor samples, and statistical significance of DEGs was presented by calculating a P-value according to Poisson distribution. Then, significance of a DEG/transcript between two samples was determined according the threshold of llog2 (fold change) $\mid>5$ and false discovery rate (FDR) adjusted to $\mathrm{P}<0.05$.

Kyoto Encyclopedia of Genes and Genomes (KEGG) pathway enrichment analysis of DEGs. Upregulated genes and downregulated genes identified by comparing gene expression between tumor and para-tumor samples were analyzed using the KEGG pathway database, as previously described (9), in order to determine the biological function of these DEGs. Enriched pathway was determined by significant fisher exact test $(\mathrm{P}<0.05)$, and at least 3 DEGs were involved in the pathway. The pathway enrichment analysis was performed using 'KEGG.db' and 'KEGGprofile' packages from R project.

\section{Results}

Overall gene expression profiling of iCCA samples. In order to characterize the global gene expression changes of iCCA samples, all the sequenced reads were aligned to the Ensembl GRCh38 human genome. Expression level values (FPKM) for 64,232 genes (including coding and non-coding genes) were obtained, and 20,880 genes with zero FPKM in all the 14 samples were excluded, finally, 43,352 genes remained. After $\log 2$ transformation of FPKM values, HCA revealed the variability of each sample in terms of their gene expression levels. Samples were clearly clustered into two groups according to tumor and para-tumor samples (Fig. 1). The results of HCA showed marked gene expression alteration in iCCA tumor tissues. In addition, high correlation coefficient within the tumor and para-tumor groups indicates a satisfactory reproducibility and low variability in iCCA biological samples.

DEG analysis. DEGs were identified by comparing the gene expression profiles of 7 tumor samples and matched para-tumor tissues. The same cutoffs of $\mathrm{P}<0.05$ and fold change $>2$ were applied to select DEGs for all tumor samples. As shown in
Fig. 2, the number of DEGs in 7 tumor samples varied from 45 to 115 , and 284 unique genes were identified to be DEGs in at least one iCCA patient. No overlapping DEGs were identified between the 7 iCCA samples. In addition, except for the iCCA4 sample, the number of downregulated genes was at least double that of the upregulated genes in each sample. This may indicate an overall downregulation transcriptomic expression in iCCA tumors. Differential expression of genes specific to iCCA were identified as deregulated genes in $\geq 4$ of the iCCA samples.

Pathway enrichment analysis of DEGs. To further clarify the biological function of identified DEGs, pathway enrichment analysis was performed by analyzing the DEGs using the KEGG pathway database. Pathways with a fisher's exact $\mathrm{P}<0.05$ and with at least three genes involved were determined to be significantly enriched. The results of the enriched pathways of the 140 downregulated genes are listed in Table I. The top enriched pathway was associated with fat digestion and absorption; other pathways were associated with the basic biological processes of the liver and gallbladder. The downregulation of these key metabolism genes may indicate the loss of these functions in iCCA tumors.

Following analysis of the 107 upregulated genes, only three pathways were enriched (Table II). The top enriched pathway was salivary secretion, and no metabolic pathways were identified to be enriched. Notably, 4 upregulated genes were assigned to the significantly enriched Wnt signaling pathway and cytokine-cytokine receptor interaction pathway, respectively. The relevance of these pathways in cholangiocarcinoma or hepatocellular carcinoma has previously been confirmed by several studies $(10,11)$.

Identification of iCCA specific DEGs. Whole-transcriptome sequencing allows the determination of key alterations in gene expression profiles that are associated with the pathogenesis of iCCA. As shown in Fig. 3, genes which were differentially expressed in at least 4 tumor samples were identified as specifically upregulated or downregulated in iCCA. The genes PDZK1IP1 (also termed MAP17), RPL41 and EEF1A2 were significantly upregulated in iCCA tumor samples.

Furthermore, several genes associated with the immune system and metabolism were significantly downregulated (Table III). MBL2, ORM2, C8A and CD5L are important elements in the innate immune system. The downregulation of these genes may indicate a defect in immune function contributing to the tumorigenesis of iCCA. LPA, APOF, HAO1, SLC10A1, GYS2 and SULT2A1 involved in the lipid metabolism and circulation of bile acids were also found to be downregulated in iCCA. Furthermore, genes involved in metabolic energy and glycogen synthesis, such as GYS2, ABCG8 and NUGGC are negatively regulated in iCCA tumor tissues. Thus, the basic biological functions of the liver, including glycogen synthesis, are influenced by iCCA tumors.

\section{Discussion}

iCCA is an aggressive primary liver tumor arising from the epithelial cells of intrahepatic bile ducts and the incidence of this disease has increased over the past two decades (2). Dioxin 
Table I. Enriched pathways of downregulated genes.

\begin{tabular}{lcccc}
\hline Pathway & DEGs & Genes & DEGs/Genes (\%) & P-value \\
\hline Fat digestion and absorption & 8 & 46 & 0.17 & $6.65 \mathrm{E}-10$ \\
Bile secretion & 8 & 71 & 0.11 & $3.54 \mathrm{E}-08$ \\
Caffeine metabolism & 3 & 7 & 0.43 & $3.79 \mathrm{E}-07$ \\
Steroid hormone biosynthesis & 5 & 57 & 0.09 & $2.41 \mathrm{E}-05$ \\
Complement and coagulation cascades & 5 & 69 & 0.07 & $7.14 \mathrm{E}-05$ \\
Metabolism of xenobiotics by cytochrome P450 & 5 & 71 & 0.07 & $8.38 \mathrm{E}-05$ \\
Drug metabolism-cytochrome P450 & 5 & 73 & 0.07 & $9.79 \mathrm{E}-05$ \\
Linoleic acid metabolism & 3 & 30 & 0.1 & $2.44 \mathrm{E}-04$ \\
Retinol metabolism & 4 & 64 & 0.06 & $4.95 \mathrm{E}-04$ \\
ABC transporters & 3 & 44 & 0.07 & $1.07 \mathrm{E}-03$ \\
Drug metabolism-other enzymes & 3 & 52 & 0.06 & $2.00 \mathrm{E}-03$ \\
Starch and sucrose metabolism & 3 & 54 & 0.06 & $2.30 \mathrm{E}-03$ \\
Pancreatic secretion & 4 & 101 & 0.04 & $3.73 \mathrm{E}-03$ \\
Cytokine-cytokine receptor interaction & 7 & 265 & 0.03 & $4.78 \mathrm{E}-03$ \\
PPAR signaling pathway & 3 & 70 & 0.04 & $5.83 \mathrm{E}-03$ \\
Vascular smooth muscle contraction & 4 & 116 & 0.03 & $6.64 \mathrm{E}-03$
\end{tabular}

DEGs, differentially expressed genes.

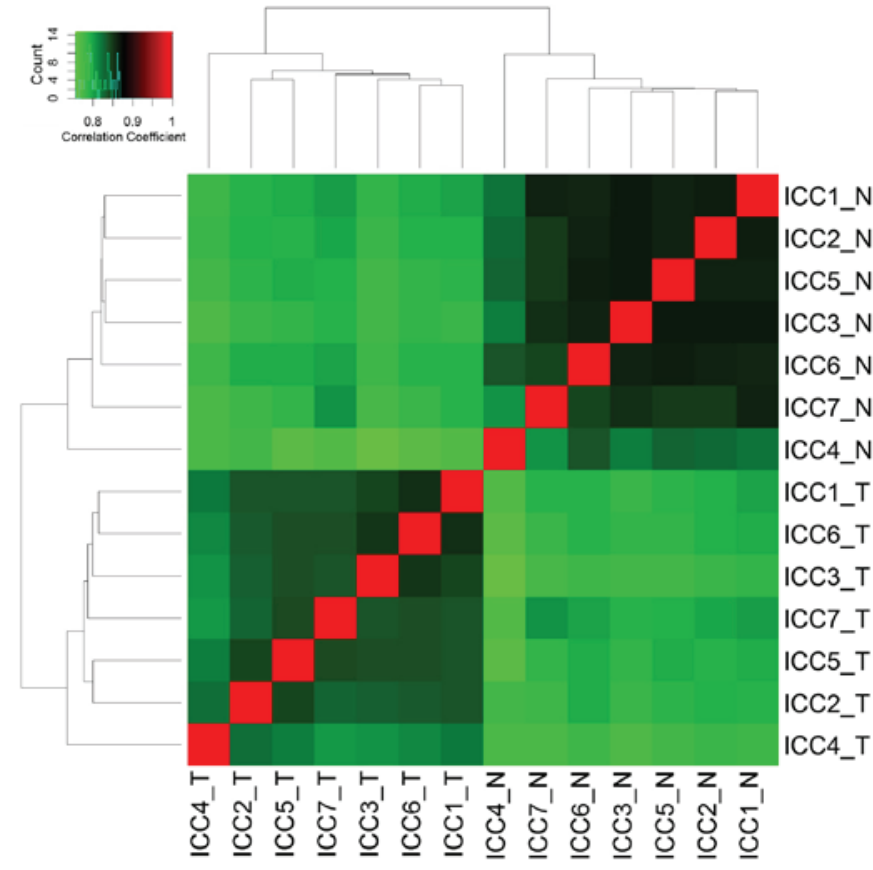

Figure 1. Hierarchical clustering analysis and heatmap of the correlation coefficients between the seven gene expression profiles of intrahepatic cholangiocarcinoma. 'T' represents tumor samples, and 'N' represents para-tumor samples. Green-red indicates correlation coefficients from low to high, respectively.

exposure, inflammatory disease, and parasitic liver diseases may be predominant risk factors of iCCA. However, the molecular mechanism of iCCA tumorigenesis remains unclear. The genome-wide gene expression of iCCA was investigated in order to demonstrate the molecular pathogenesis of iCCA. The expression of 43,352 genes was quantified using RNA-Seq data.

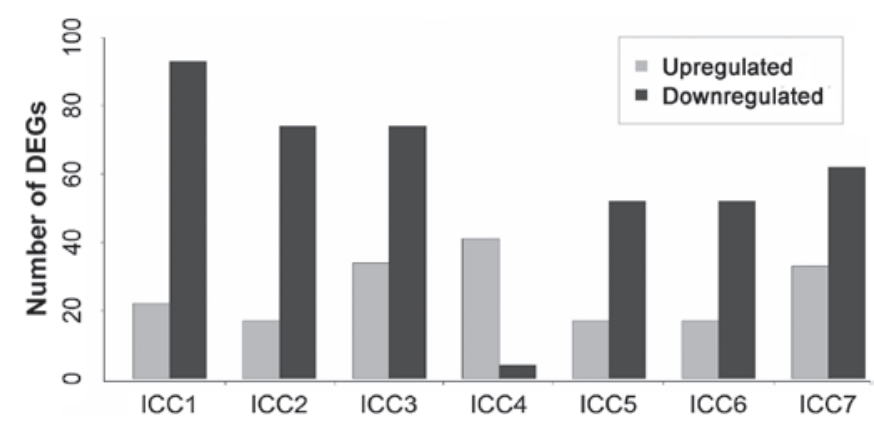

Figure 2. Number of DEGs in each of the seven iCCA samples. The absolute number of differentially expressed genes are shown on the $y$-axis and the seven iCCA samples are shown on the $\mathrm{x}$-axis. DEGs, differentially expressed genes; iCCA, intrahepatic cholangiocarcinoma.

Differential gene expression analysis and functional annotation tools were employed to clarify the changes in expression profiles, and further demonstrated the molecular mechanism underlying the tumorigenesis and progression of iCCA. In this study, PDZK1IP1, RPL41 and EEF1A2 were identified to be significantly overexpressed in iCCA.

RPL41, which is a novel protein coding gene annotated in the GRCh38 human genome database (12), was upregulated in the present study. To the best of our knowledge, this is the first annotation of RPL41 overexpression in iCCA. As a small ribosomal peptide deregulated in tumors, RPL41 is essential for mitosis and centrosome integrity (13), and also functions as a tumor suppressor and deregulates the bioactivity of tumor cells $(13,14)$. The correlation between RPL41 and iCCA requires further investigation.

PDZK1IP1, PDZK1-interacting protein 1, is a small $17 \mathrm{kDa}$ non-glycosylated membrane protein, researchers have revealed the overexpression of PDZK1IP1 in a variety of 
Table II. Enriched pathways of upregulated genes.

\begin{tabular}{|c|c|c|c|c|}
\hline Pathway & DEGs & Genes & DEGs/Genes (\%) & P-value \\
\hline Salivary secretion & 4 & 89 & 0.04 & 8.17E-05 \\
\hline Wnt signaling pathway & 4 & 151 & 0.03 & $9.18 \mathrm{E}-04$ \\
\hline Cytokine-cytokine receptor interaction & 4 & 265 & 0.02 & $9.65 \mathrm{E}-03$ \\
\hline
\end{tabular}

DEGs, differentially expressed genes.

Table III. Gene ontology annotation of key intrahepatic cholangiocarcinoma-related genes.

\begin{tabular}{llll}
\hline Category & \multicolumn{1}{c}{ Term } & \multicolumn{1}{c}{ Term name } & \multicolumn{1}{c}{ Genes } \\
\hline Immune $\downarrow$ & GO:0006950 & Response to stress & C8A, MBL2, LPA, F9, CD5L, ORM2 \\
GO:0006952 & Defense response & C8A, MBL2, CD5L, ORM2 \\
GO:0006954 & Inflammatory response & C8A, MBL2, ORM2 \\
GO:0006629 & Lipid metabolic process & HAO1, LPA, SULT2A1, APOF \\
GO:0002682 & Regulation of immune & C8A, MBL2, ORM2 \\
Metabolism $\downarrow$ & system process & ABCG8, LPA, APOF \\
& GO:0006869 & Lipid transport & ABCG8, SULT2A1 \\
GO:0007586 & Digestion & HAO1, SULT2A1 \\
Cancer $\downarrow$ & GO:0006460xylic acid catabolic & RPL41, EEF1A2 \\
\hline
\end{tabular}

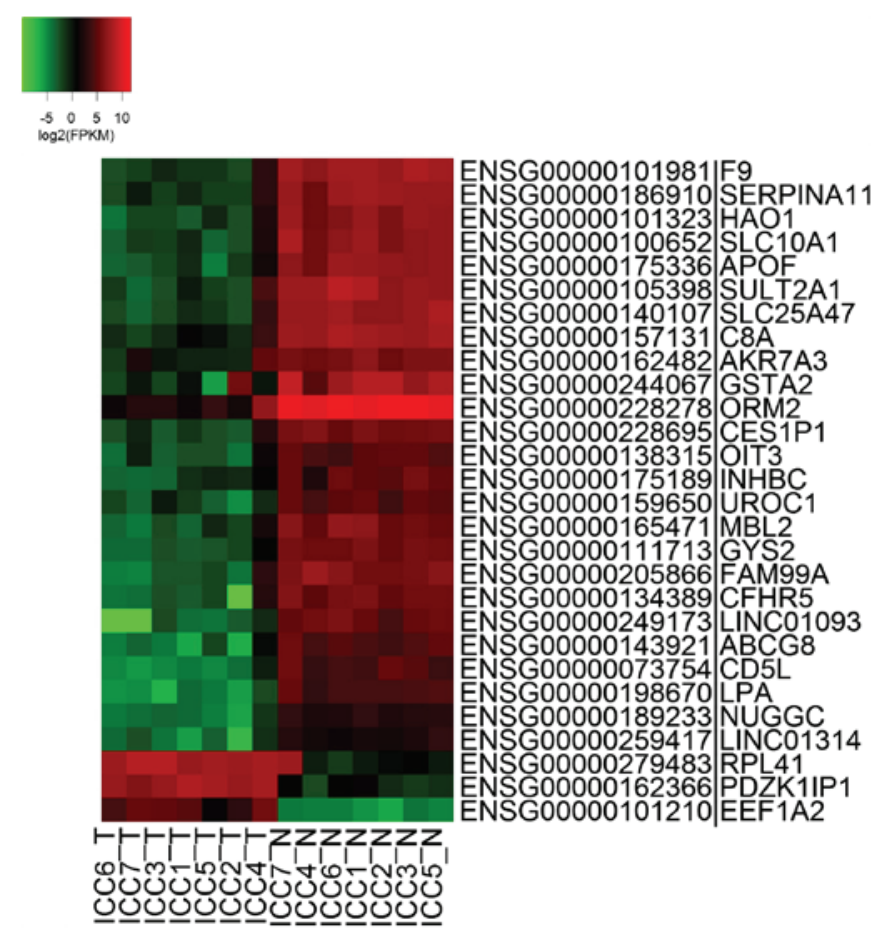

Figure 3. Heatmap for genes differentially expressed in at least 4 iCCA tumor samples. iCCA, intrahepatic cholangiocarcinoma.

human carcinomas (15). As a common characteristic of carcinoma (15) overexpression of PDZK1IP1 enhances malignant behavior such as aggression and invasion of tumor cells (16).
Overexpression of this gene was also observed in the present study, demonstrating its important role in the progression of iCCA. Upregulation of PDZK1IP1 has been demonstrated to enhance reactive oxygen species (ROS) production and tumorigenesis (16) as well as inhibit Myc-induced apoptosis through PI3K/AKT pathway activation (17). Therefore, PDZK1IP1 may be a key element in the tumorigenesis and progression of iCCA and may be associated with a poor prognosis.

EEF1A2, Elongation factor 1- $\alpha 2$, is upregulated in numerous cancer types and is essential for cell migration, invasion and metastasis in cancer $(18,19)$. As a translation elongation factor, EEF1A2 binds to amino acylated tRNA directly and guides its connection with the ribosome and mRNA codon (20). Previous studies have identified the overexpression of EEF1A2 in various tumors, including breast cancer, ovarian cancer and prostate cancer (18,21-23). In addition, it was demonstrated that the upregulation of EEF1A2 can promote cancer cell migration, invasion and metastasis (19); therefore, as the present study identified EEF1A2 overexpression in iCCA, EEF1A2 may be a useful prognostic factor for iCCA.

In addition, the SLC25A47 gene is significantly downregulated in iCCA, and its encoded protein has the hallmark features of mitochondrial carrier proteins in hepatocellular carcinoma (24). Furthermore, downregulation of a solute carrier family gene SLC25A47 (also termed HDMCP) and a serpin peptidase inhibitor SERPINA11 was also associated with iCCA pathogenesis. Consistently, downregulation of SLC25A47 has been shown to induce the dissipation of the mitochondrial membrane potential in hepatocellular carcinoma, a common phenomenon observed in cancer cells (24). 
SERPINA11 is correlated with pathological stage of hepatocellular carcinoma, and is downregulated in oral squamous cell carcinomas (25). Thus, these genes could be a potential therapeutic target and biomarker in the treatment of iCCA.

Overall expression profiling identified a difference between para-tumor and tumor tissues, however, consistency was observed among the profiles of tumor tissues from different individuals. In total, 164 downregulated genes and 121 upregulated genes were identified in at least one tumor sample. KEGG pathway enrichment analysis of the DEGs demonstrated that dysregulated genes were predominantly associated with fat digestion and absorption, bile secretion and other metabolism pathways, which may indicate that the basic biological functions of the liver and cholangiole were altered in iCCA. In addition, 4 genes associated with the Wnt signaling pathway were significantly upregulated. Thus tumorigenesis and progression of iCCA may lead to the dysfunction of liver metabolism. Consistent with the present results, the expression of the SLC10A1 gene (a member of the sodium/bile acid cotransporter family which participates in the enterohepatic circulation of bile acids) was decreased in cholangiocarcinoma (26). SULT2A1, encoding a protein which catalyzes the sulfation of steroids and bile acids in the liver and adrenal glands, and the ABCG8 gene, which is associated with cholesterol absorption, were downregulated in iCCA. SULT2A1 and ABCG8 are important role in the bile secretion pathway. LPA (also termed APOA) and APOF involved in transport and/or esterification of cholesterol were also decreased in iCCA. This may indicate the dysfunction in lipid metabolism and the influence of iCCA on the biological functions of the liver.

In conclusion, PDZK1IP1, RPL41 and EEF1A2 were significantly upregulated in iCCA and may be associated with the poor prognosis of iCCA. Metabolism and immune function-related genes, MBL2, ORM2, C8A, CD5L and LPA, APOF, HAO1, SLC10A1, GYS2 were significantly downregulated and influenced in iCCA patients respectively. Therefore, the present study elucidated the gene expression patterns associated with the tumorigenesis of iCCA, which may be novel diagnostic factors for iCCA

\section{Acknowledgements}

This study was funded by the Outstanding Leaders Training Program of Pudong Health Bureau of Shanghai (grant no. PWR12015-04) and the Scientific Research Start-up Funding of the Seventh People's Hospital Affiliated to Shanghai University of Traditional Chinese Medicine.

\section{References}

1. Bosman FT, Carneiro F, Hruban RH and Theise ND: WHO classification of tumours of the digestive system. World Health Organization, 2010.

2. Everhart JE and Ruhl CE: Burden of digestive diseases in the United States part II: Lower gastrointestinal diseases. Gastroenterology 136: 741-754, 2009

3. Wang AG, Yoon SY, Oh JH, Jeon YJ, Kim M, Kim JM, Byun SS, Yang JO, Kim JH, Kim DG, et al: Identification of intrahepatic cholangiocarcinoma related genes by comparison with normal liver tissues using expressed sequence tags. Biochem Biophys Res Commun 345: 1022-1032, 2006.

4. Obama K, Ura K, Li M, Katagiri T, Tsunoda T, Nomura A, Satoh S, Nakamura Y and Furukawa Y: Genome-wide analysis of gene expression in human intrahepatic cholangiocarcinoma. Hepatology 41: 1339-1348, 2005.
5. Sia D, Losic B, Moeini A, Cabellos L, Hao K, Revill K, Bonal D, Miltiadous O, Zhang Z, Hoshida Y, et al: Massive parallel sequencing uncovers actionable FGFR2-PPHLN1 fusion and ARAF mutations in intrahepatic cholangiocarcinoma. Nat Commun 6: 6087, 2015.

6. Kim D, Pertea G, Trapnell C, Pimentel H, Kelley R and Salzberg SL: TopHat2: Accurate alignment of transcriptomes in the presence of insertions, deletions and gene fusions. Genome Biology 14: R36, 2013.

7. Trapnell C, Roberts A, Goff L, Pertea G, Kim D, Kelley DR, Pimentel H, Salzberg SL, Rinn JL and Pachter L: Differential gene and transcript expression analysis of RNA-seq experiments with TopHat and Cufflinks. Nat Protoc 7: 562-578, 2012.

8. Ward JH Jr: Hierarchical grouping to optimize an objective function. J Am Statist Assoc 58: 236-244. 1963.

9. Kanehisa M and Goto S: KEGG: Kyoto encyclopedia of genes and genomes. Nucleic Acids Res 28: 27-30,2000.

10. Patel T: New insights into the molecular pathogenesis of intrahepatic cholangiocarcinoma. J Gastroenterol 49: 165-172, 2014.

11. DeMorrow S, Francis H, Gaudio E, Venter J, Franchitto A, Kopriva S, Onori P, Mancinelli R, Frampton G, Coufal M and Mitchell B: The endocannabinoid anandamide inhibits cholangiocarcinoma growth via activation of the noncanonical Wnt signaling pathway. Am J Physiol Gastrointest Liver Physiol 295: G1150-G1158, 2008.

12. Cunningham F, Amode MR, Barrell D, Beal K, Billis K, Brent S, Carvalho-Silva D, Clapham P, Coates G, Fitzgerald S, et al: Ensembl 2015. Nucleic Acids Res 43 (Database issue): D662-D669, 2015.

13. Wang S, Huang J, He J, Wang A, Xu S, Huang SF and Xiao S: RPL41, a small ribosomal peptide deregulated in tumors, is essential for mitosis and centrosome integrity. Neoplasia 12: 284-293, 2010

14. Wang A, Xu S, Zhang X, He J, Yan D, Yang Z and Xiao S: Ribosomal protein RPL41 induces rapid degradation of ATF4, a transcription factor critical for tumour cell survival in stress. J Pathol 225: 285-292, 2011.

15. Guijarro MV, Leal JF, Fominaya J, Lleonart M, Castellvi J, Ruiz L, Ramon Y Cajal S and Carnero A: MAP17 overexpression is a common characteristic of carcinomas. Carcinogenesis 28: 1646-1652, 2007.

16. Guijarro MV, Leal JF, Blanco-Aparicio C, Alonso S, Fominaya J, Lleonart M, Castellvi J, Ramon y Cajal S and Carnero A: MAP17 enhances the malignant behavior of tumor cells through ROS increase. Carcinogenesis 28: 2096-2104, 2007.

17. Guijarro MV, Link W, Rosado A, Leal JF and Carnero A: MAP17 inhibits Myc-induced apoptosis through PI3K/AKT pathway activation. Carcinogenesis 28: 2443-2450, 2007.

18. Sun Y, Du C, Wang B, Zhang Y, Liu X and Ren G: Up-regulation of eEF1A2 promotes proliferation and inhibits apoptosis in prostate cancer. Biochem Biophys Res Commun 450: 1-6, 2014.

19. Xu C, Hu DM and Zhu Q: eEF1A2 promotes cell migration, invasion and metastasis in pancreatic cancer by upregulating MMP-9 expression through Akt activation. Clin Exp Metastasis 30: 933-944, 2013.

20. Browne GJ and Proud CG: Regulation of peptide-chain elongation in mammalian cells. Eur J Biochem 269: 5360-5368, 2002.

21. Tomlinson VA, Newbery HJ, Wray NR, Jackson J, Larionov A, Miller WR, Dixon JM and Abbott CM: Translation elongation factor eEF1A2 is a potential oncoprotein that is overexpressed in two-thirds of breast tumours. BMC Cancer 5: 113, 2005.

22. Kulkarni G, Turbin DA, Amiri A, Jeganathan S, Andrade-Navarro MA, Wu TD, Huntsman DG and Lee JM: Expression of protein elongation factor eEF1A2 predicts favorable outcome in breast cancer. Breast Cancer Res Treat 102: 31-41, 2007.

23. Pinke DE, Kalloger SE, Francetic T, Huntsman DG and Lee JM: The prognostic significance of elongation factor eEF1A2 in ovarian cancer. Gynecol Oncol 108: 561-8, 2008.

24. Tan MG, Ooi LL, Aw SE and Hui KM: Cloning and identification of hepatocellular carcinoma down-regulated mitochondrial carrier protein, a novel liver-specific uncoupling protein. J Biol Chem 279: 45235-45244, 2004.

25. Shiiba M, Nomura H, Shinozuka K, Saito K, Kouzu Y, Kasamatsu A, Sakamoto Y, Murano A, Ono K, Ogawara K, et al: Down-regulated expression of SERPIN genes located on chromosome 18q21 in oral squamous cell carcinomas. Oncol Rep 24: 241-249, 2010.

26. Yeh CN, Weng WH, Lenka G, Tsao LC, Chiang KC, Pang ST, Chen TW, Jan YY and Chen MF: cDNA microarray profiling of rat cholangiocarcinoma induced by thioacetamide. Mol Med Rep 8: 350-360, 2013. 